\title{
Trailing AIDS in Central Africa
}

\section{A meeting in Senegal enabled AIDS researchers to learn more of the possible African origin of the virus that causes AIDS and virus-associated cancers in Africa.}

BECAUSE of growing evidence that the virus that causes acquired immune deficiency syndrome (AIDS) originated in Central Africa, AIDS researchers are turning to their atlases. Earlier this month, on 8-10 May, some landed there for the first time to attend a meeting arranged by the French Association pour la Recherche sur le Cancer at the faculty of medicine in Dakar, Senegal, on virus-associated cancers in Africa. What they were forcibly reminded of is that in African terms, AIDS is an insignificant problem, but that most common forms of cancer in Africa are caused in part by viruses.

At times the question of the origin of AIDS has seemed to be no more than a matter of geographical buck-passing first between the United States and Haiti and then to and within Africa - but the issue is much more serious. At its worst, the argument is that the virus (HTLV-III or LAV) has only recently emerged in virulent form, has been able to do so as a result of changing socio-economic factors in Africa and may be followed by equivalent conversions to virulence of other relatively harmless viruses. More optimistically, the question becomes: can one learn anything from the origin of the virus that would help to contain the disease?

Geographically, the finger of suspicion points at Central Africa, possibly Zaire, as the location from which the virus has spread. The data, however, are sketchy and dependent on what historical and contemporary sera samples are available for analysis, and to whom. From one of the most extensive surveys so far, G.de The of the CNRS, Lyon, provisionally concluded that the virus emerged in Kenya only after 1970, that there are still very puzzling differences between adjacent countries, and that there may be evidence of change of viral form in some comparative data.

The evidence of change comes from the comparison of two tests, one of which (an enzyme-linked immunosorbent assay) is less sensitive to change than the other ("Western blotting" of proteins). That the current virus is very prone to change is clear. The sequences of viruses that came directly or indirectly from patients in New York in 1982-83 differ by only a few per cent, whereas the sequences of a virus from a Haitian taken in the same period and one from a resident of San Francisco isolated in 1984 show considerable differences. Most variation is in the mid-portion of the gene that encodes the envelope protein of the virus. Up to 25 per cent variation is recorded there, according to $W$. Haseltine of Harvard Medical School.

By definition, however, all of these isolates and the other hundred or so whose variation is fully documented in only a few cases, are from AIDS patients. All, therefore, have caused immunodeficiency, and no correlation has emerged between variation in viral structure and the precise manifestations of the immunodeficiency syndromes. The pressing question is whether such a correlation can be found and, in particular, whether the African population will yield variants that are less prone to cause AIDS and from which the "American" variants are descended. In other words, do viral differences account for the fact that AIDS in Central Africa seems to be equally distributed between the sexes and transmitted by heterosexual activities rather than largely by homosexual practice and blood or its products? Or are these differences only a temporary reflection of the initial entry of the virus into the industrialized nations through the homosexual community? The signs are more that the latter is the case.

Now the focus of attention has shifted to the African green monkey. Most of some recently collected specimens from the wild in Zaire contain serum proteins characteristic of HTLV-III/LAV and yet are healthy, according to M. Essex from Harvard School of Public Health. A comparative study of the monkey and human virus together with that which caused an outbreak of AIDS-like disease in a captive colony of Macaque monkeys in the New England Primate Center may help to clarify the origin and virulence of HTLV-III/LAV and suggest approaches to vaccination or therapy.

A separate reason for delving deeply into variants is to discover whether sequence changes are responsible for some of the human pathologies other than AIDS with which HTLV-III/LAV is coming to be associated. R. Gallo from the National Cancer Institute in Bethesda, Maryland, pointed in particular to an association with brain abnormalities in children without immune deficiency; evidence exists for the presence of replicating virus in the human brain, particularly in glial cells. Such evidence tends to strengthen the claims of both Gallo and S. Wain-Hobson, of the Institut Pasteur in Paris, that HTLVIII/LAV is related to the lentiviruses, of which the prototype is visna virus, which causes a slowly progressive demyelinating disease of the central nervous system of sheep. Gallo also tentatively suggested a new pathological involvement of the virus that would dispense with the first word of idiopathic thrombocytopaenic purpura.

While AIDS is a growing concern in the industrialized nations, African priorities lie elsewhere. In Senegal, for example, either there is no AIDS or it has not yet been recognized, whereas many die from a liver cancer caused in part by hepatitis B virus. In Africa generally this, and cervical cancer are major types of cancer. A third virusassociated cancer in Africa is Burkitt's lymphoma, an infrequent disease but responsible for many childhood tumours and causally linked to the Epstein-Barr virus.

None of these viruses causes cancer without contributory factors, of which immunodeficiency is the most interesting to AIDS researchers. For Burkitt's lymphoma, immonodeficiency - in the form of lost of T-lymphocyte control of EpsteinBarr virus infected $B$ cells - is induced by malaria, as shown by $\mathrm{H}$. Whittle of the MRC Laboratories, The Gambia. And a mini-epidemic of Burkitt's lymphoma in US AIDS patients suggests that any form of immunosuppression is liable to activate the latent virus.

Hopes, however thin, are pinned on the development of a vaccine against HTLVIII/LAV. For the hepatitis B virus, vaccines are already in trial but for the EpsteinBarr virus the first glimpse of a vaccine was provided in Senegal by M.A. Epstein of the University of Bristol. The vaccine is composed of one of the major proteins of the virus and protects cotton-top tamarins against cancers induced by the virus. The tamarin disease does not altogether resemble the human disease and the frequency of Burkitt's lymphoma may never warrant mass vaccination in terms of cost. In Asian countries where the virus is a part cause of nasopharyngeal cancer, vaccination may be more realistic. Epstein foresees an initial trial of the vaccine against mononucleosis, the relatively mild result of infection by the virus after childhood.

The AIDS researchers who dropped in on Senegal need African collaborators in order, at the least, to carry out extensive trans-African surveys of AIDS, its virus and its origins. The Africans leaving Senegal knew at least that they had increased the awareness of the diseases and priorities in Africa among those who could provide help. 